Voix et Images

volxetimages

\title{
Marie-Claire Blais : "Je veux aller le plus loin possible ». Entrevue
}

\section{Gilles Marcotte}

Volume 8, numéro 2, hiver 1983

Marie-Claire Blais

URI : https://id.erudit.org/iderudit/200380ar

DOI : https://doi.org/10.7202/200380ar

Aller au sommaire du numéro

Éditeur(s)

Les Presses de l’Université du Québec

ISSN

0318-9201 (imprimé)

1705-933X (numérique)

Découvrir la revue

Citer ce document

Marcotte, G. (1983). Marie-Claire Blais : « Je veux aller le plus loin possible ».

Entrevue. Voix et Images, 8(2), 191-209. https://doi.org/10.7202/200380ar d'utilisation que vous pouvez consulter en ligne.

https://apropos.erudit.org/fr/usagers/politique-dutilisation/ 


\title{
Marie-Claire Blais: "Je veux aller le plus loin possible»
}

\author{
Entrevue/Gilles Marcotte, Université de Montréal
}

V.1.: Depuis que je vous lis, j'ai toujours eu l'impression que vous vous faisiez une idée très ferme, très haute, du rôle - il faudrait peut-être dire la vocation - de l'écrivain, semblable à celle qui soutient Jean-Le Maigre ou Pauline Archange. Pour celle-ci, par exemple, l'écriture est une question d'existence, une question de vie ou de mort, comme elle le dit a la fin du roman: asans ces quelques pages, je risquais de n'avoir existé pour personnen. La vie est vide, inutile, si elle n'est pas dite, travaillée, refaite par l’écriture. Et cela n'est pas vrai seulement pour la personne qui écrit. mais aussi pour le monde qui l'entoure, dont elle témoigne. Pensez-vous que l'écriture a pour mission de sauver la vie?

M.-C. B.: Je pense que c'est peut-être faux de penser comme je pensais autrefois - je pense pas ca aujourd'hui - que la vie est vide. inutile. si on n'écrit pas; parce que la plupart des gens n'écrivent pas et ils vivent des vies extrêmement utiles, peut-être plus utiles que la mienne, et fécondes. Mais je pense que pour moi... C'est surtout très personnel d'avoir écrit ca, c'est le témoignage de ma vie, l'écriture, c'est comme ca que je la ressens. Mais, naturellement, c'est sûr que toutes les vies sont des témoignages vivants. Je pense que c'est fort arrogant d'avoir dit ca...

V.I.: Est-ce quion ne pourrait pas dire que pour vous l'écrivain est en quelque sorte le délégué des autres, de ceux qui n'écrivent pas? C'est celui qui porte à l'écriture les vies, l'ensemble des vies qui l'entourent, et qui, elles, ne peuvent pas accéder directement à l'écriture.

M.-C. B.: Il contient beaucoup de vies en lui, dans son témoignage. Puis il parle pour les autres, parce qu'il voit les autres, il est le visionnaire des autres, le visionnaire de son temps, témoin de son temps. Mais j'ai de plus en plus de doutes sur sa... sur sa vocation. Plus j'écris, plus j'ai des doutes. Alors il me semble que ces certitudes que j'avais, disons très jeune. je commence a les avoir un peu moins. 
V.I.: Est-ce que vous pourriez précıser ces doutes que vous avez?

M.-C. B.: Je viens de lire le témoıgnage très émouvant de Romain Gary, que vous avez sans doute lu, sur Émile Ajar et ce rôle double qu'ils ont joué ensemble. Gary et lui-même. II raconte I'historre d'un métier qui dans ce siècle, parmi les gens qu'il connait, les milieux qu'il connait, les milieux littéraires français, il dit que ce métier n'a presque plus de fonction. il parle de fonction lyrıque. Ca m'a énormément touchée - c'est un tout petit "pamphlet - ca m'a énormément touchée que cet homme - évidemment, on peut tout dire quand on va mourır - qu'il livre un message aussi dur, mais aussi réel, aussi tangible pour moi. Pour moi, c'est bien réel que, en ce moment, pour nous, pour les autres... est-ce que c'est important d'écrire encore? Moi, je le fais parce que j'aime mon métier, c'est ma seule vie, et lui. il l'a fait parce que c'était sa seule vie, quitte à changer de rôle. Mais il $\gamma$ a dans ce epamphlet» beaucoup d'interrogations qui me sont proches.

V.I.: Et pourtant en lisant votre avant-dernier livre, votre avant-dernier roman, le Sourd dans la ville. j'ai l'impression que malgré tous les doutes que vous pouvez avoir, de l'extérieur pour ainsi dire, quand vous entrez en contact avec l'écriture il y a là pour vous quelque chose d'absolument nécessaire qui ne souffre pas d'être remis en question... personnellement.

M.-C. B.: Peut-être que c'est ce qui arrive aussi... Quand on est dans certe espèce de noce intime, là, entre le travail et soi, on est très heureux, on sent la fécondité. Mais c'est quand on arrive, quand on lance tout ca dans le vide d'aujourd'hui... II y a une espèce de vide de pensée qu'il n'y avait pas au début du siècle. enfin au temps où on n'était pas là. Et pour l'écrivain... J'imagine qu'un jeune écrivain de vingt ans, aujourd'hui, aura énormément plus de problèmes que nous en avons eu.

Les apprentissages

Ecrire un roman, c'est savoir que chaque mot fait aussi partie d'un immense trésor. Oh! si un jour je pouvais écrire des choses belles I Ne pas écrire le désespoir, mais l'espérance. Je me réjouis de sentir que chaque mot a une musique. J'écris aforèt * et la forêt sort de mes veines. J'écris "nuit" et mes yeux connaissent le noir du sommeil.

\section{Tête blanche}

V.I.: Mais vous, justement, quand vous avez commencé à écrire... Je me souviens d'une liasse de manuscrits, poèmes, récits que Jeanne Lapointe nous lisait entre deux séances de I'Institut canadien des affaires publiques, à Sainte-Adèle je crois, à la fin des années 
cinquante. Comme vous $y$ alliez! D'où cela vous venait-il? Vous n'aviez pas vécu, si je ne me trompe, dans un milieu particulièrement propice à la lecture et à la création littéraire.

M.-C. B. : Non, pas du tout. Mais ca arrive qu'on soit comme ca expulsé dans l'écriture... par amour, par fièvre. Et j'ai eu la chance justement. comme vous nommez Madame Lapointe, de connaitre, d'avorr connu à cette époque - moi, j'avais beaucoup de feu et beaucoup de brouilIon dans mes idées - quelqu'un de sévère, d'austère, pour quasıment me donner l'aliment de ma pensée, qui n'était pas claire à l'époque. C'est normal quand on est très jeune. Mais je ne suis pas sûre quaujourd'hui les jeunes écrivains rencontrent quelqu'un pour les aider comme ca. J'ai eu énormément de chance.

V.I.: Nous parlons de Jeanne Lapointe, qui est professeur a l'Université - Laval. Est-ce dire que vous avez fait des études littéraires, a proprement parler?

M.-C. B.: Non. J'étudiais de temps en temps, mais je travaillais, je gagnais ma vie, à l'époque. C'était un temps où l'on pouvait gagner sa vie très jeune.

V.I.: Et le Père Georges-Henri Lévesque, il a eu lui aussi une grande importance pour vous, à la même époque.

M. -C. B.: Oui, c'était un homme de grande qualité qui s'intéressait à des êtres marginaux. Parce qu'à l'époque, il faut penser que les artistes étaient des êtres marginaux quand même. Je pense que cette espèce d'amour de l'art, des artistes, qu'il y avait, nous l'avons beaucoup moins aujourd'hui. Et beaucoup d'artistes vont se perdre à cause de ca, dans un néaǹt de pensée, dans une pensée qui n'aura pas la rigueur que nous avons connue, très jeunes, parce que le Père Lévesque, Madame Lapointe, c'étaient des gens qui inspiraient la rigueur. Et il fallait ça pour un écrivain incendié d'idées!

V.I.: Je vais vous poser une question qui vous paraîtra peut-être indiscrète. Le milieu dans lequel vous viviez dans ce temps-là, est-ce qu'il était un peu celui de Pauline Archange?

M.-C. B.: Peut-être un peu. Disons qu'il n'y avait pas d'assistance intellectuelle. sur ce plan-là, comme dans Pauline Archange. Mais il y avait le même amour qu'il y a dans Pauline Archange, beaucoup d'attention des parents et beaucoup d'affection familiale. Naturellement, si vous avez plusieurs enfants dans une famille... Le problème de l'artiste est un peu, alors, ce qu'il deviendra plus tard - c'est-à-dire que dans une collectivité. l'artiste n'a pas tellement de place. Les parents qui ont plusieurs enfants n'ont pas le temps de ne s'occuper que d'un seul.

V.I.: II y a aussi dans Pauline Archange un petit milieu littéraire, avec Romaine Petit-Page et les autres. Est-ce que vous avez connu quelque chose de semblable? 
M.-C.B.: Oui, et très jeune. Vers l'âge de quinze ans, j’al connu un milieu comme ça, que peut-ètre vous avez connu aussi, à Québec. Enfin, Québec, ça ne se compare à aucune ville... sauf à des villes françaises, maıs il y avait un mılieu littéraire, un petit milieu littéraire... provincial, à Québec. Qui était très touchant d'ailleurs, et qu'on revolt, que je revois avec attendrissement, parce que je me dis que les gens qui se débattaient pour avoir des idées, à l'époque, il leur fallait beaucoup de courage... surtout à Québec.

V.I.: Je ne. relève pas les derniers mots?... En exergue à votre premier roman, la Belle Bête, vous citez la romancière anglaise Rosamond Lehmann. Ce n'était pas un nom très répandu au Québec, à l'époque. il me semble - il ne l'est pas plus maintenant d'ailleurs. Certe référence implique-t-elle que vous aviez, dès ce moment, un bagage littéraire assez différent de celui du jeune Canadien français a cultivé o de ces années-là: Sartre, Camus, Simone de Beauvoir, Gide, un peu de Claudel, de Mauriac, de Bernanos... Que lisiez-vous?

M. C. B.: C'était une époque où je lisais tout. Vous avez dans les ceuvres de Sartre des autodidactes très jeunes qui lisent tout dans les bibliothèques, des rats de bibliothèques. Je pense que j'étais ca. Quand on n'a rien, quand on est très ignorant, quand on se sent dépossédé. on avale tout, on prend tout, on est très avide, on ne juge pas ce qu'on lit. Évidemment, moi, je lisais tout ce que je voyais, tout ce que je trouvais.

V.I.: Vous étiez vorace !

M. C. B.: Oui. Par ignorance aussi, par besoin de combler mon ignorance.

V.I.: Mais vous alliez dans des choses assez diverses, me semble-t-il, qui ne correspondaient pas toujours à ce qu'on lisait dans le milieu.

M.-C. B.: Mais les autodidactes de Sartre sont des êtres qui vont partout. IIs prennent un rayon de biblothèque, un autre, moi c'est comme ca que je lisais. J'avais une énorme avidité.

V.I.: Est-ce que vous ne lisiez pas particulièrement des cuures étrangères, qui n'étaient pas du domaine français?

M.-C. B.: Évidemment, à la bibliothèque, on avait de tout. Mais j'essayais de lire tout ce qui était étranger, oui, dans la mesure où ça me faisait partir un peu...

V.I.: Est-ce que vous avez eu, à cette époque, de grandes passions littéraires qu'il serait possible d'identifier?

M.C.B.: Très jeune, j'en avais de très grandes, comme Kafka, comme Rosamond Lehmann, que... aujourd'hui, je dirais peut-être que j'ai moins de passions, mais encore une certaine... Bien, Virginıa Woolf, ça m'avast énormément touchée, quand j'étais jeune.

V.I.: Ca vous touche sans doute encore maintenant. 
M.-C. B.: Oui, ca, je pense, avec plus de profondeur. Je veux dire, en ce temps-la on prend tout; mais au fond, est-ce qu'on absorbe tout? Mais enfin c'est très bon, parce que ca reste dans la mémoire.

Le " mal de vivre.

L'ceuvre de Camus est très importante pour les gens de ma génération puisque c'est l'œuvre abondante et merveilleuse de la recherche spirituelle, l'œuvre de la volonté de vivre, l'œuvre d'une naissance d'âme comme le réveil d'un jeune être à la vie d'homme, à des interrogations et à des tragédies d'homme.

La jeunesse prend conscience du mal de vivre et elle en est éblouie de mystère comme l'enfant inquiet qui s'enivre à sa propre respiration. Chez plusieurs de ses héros, Camus a compris cette détresse d'exister et il a apporté l'espérance d'une réponse et même parfois, un réveil de l'amour de vivre comme si l'homme, vraiment, était éternellement jeune dans sa souffrance, mélancoliquement exilé d'un infini qu'il ignore ou voudrait repousser. Ma génération est toute tendue, je crois, par les terribles problèmes de Camus. Elle cherche en lui une source reposante. Une source d'énergie et de savoir.

Inconsciemment, Camus a un peu expliqué la jeunesse dans le héros de lÉtranger qui, malheureux et tendrement avide. ne sait pas s'il a le courage de vivre ou celui de mourir...

Liberté. janvier-février 1960.

V.I.: Le seul texte que je connaisse de vous, portant sur un écrivain, c'est celui que vous avez donné à la revue Liberté, en 1960, en réponse à une enquête sur Albert Camus. Vous en parliez comme d'un témoin du a mal de vivre ....

M.-C. B.: Evidemment, toute ma génération a été très marquée par Camus. Mais aujourd'hui je n'en parlerais pas de cette façon. Peut-être aujourd'hui que ce serait beaucoup plus profond. Je prendrais plus de temps pour l'écrire.

V.I.: Pourriez-vous me donner une idée du sens quaurait aujourd'hui pour vous un témoignage sur Camus?

M.-C. B.: A l'époque on avait un a mal de vivre » mais il n'était pas, it me semble. aussi... II s'agissait de survivre, dans la pensée ou dans l'âme. après la guerre. Mais nous, aujourd'hui, on est sur un volcan; ce qu'on vit est encore plus angoissant. Donc, Camus, qui est mort très jeune, s'il était encore là, serait un témoin visionnaire d'une époque 
pas seulement décadente, mais destructrice de l'homme. Et je pense que c'est peut-ètre mieux qu'il ne l'ait pas connue... Parce que ce ce qui l'avait touché. la condition humaine qui l'avait touché. le « mal de vivre», aujourd'hui il en aurait une sorte d'épouvante, je pense...

V.l.: Pourtant l'épouvante est là, déjà, dans vos premiers romans. Je pense à la Belle Bête. Tête blanche, le Jour est noir. David Sterne. I'Insoumise. Ce sont des romans très sombres, très violents: haınes. décadences physiques et morales, et catera. Cela ne vient pas seulement, me semble-t-il, d'influences littéraires. Vous est-il possible de parler de ce amal de vivre dont vos personnages sont atteints? Dans le Sourd dans la ville, un de vos personnages est hanté par la pensée d'une sorte de cataclysme qui serait véritablement la fin du monde. L'expression a fin du monde $x$ revient souvent dans vos romans...

M.-C. B.: II faut dire que tous les personnages que vous citez, David Sterne, le a sourd « et les autres sont des gens qui sont témoins de notre siècle. On était visionnaire dans le temps de Camus et dans le temps de Mairaux, mais aujourd'hui nous avons encore un sens peut-être plus aigu du cataclysme, de tout ce qui pourrait nous emporter du jour. au lendemain. Sans lumière intellectuelle. l'esprit tend a mourir, pas à vivre, ce qui n'était pas le cas pour Camus et Sartre.

V.I.: Ce sont des gens qui prévoyaient en quelque sorte...

M.-C. B.: Ce sont de grands prophètes. Mais ces choses-là sont en nous, c'est certain que nous aussi avons à porter autant qu'eux, mais peut. être encore avec plus... d'acuité.

V.I.: La fin du monde est plus proche et plus vraie...

M.-C. B.: C'est-à-dire que nous sommes conscients, enfin nous devenons de plus en plus conscients de notre propre destruction. Nous avons encore la possibilité de dire: non, je ne veux pas... Mais laquelle? On est entouré de brutes...

V.I.: On serait tenté de parler aussi, à propos de vos premiers romans particulièrement, d'une très forte présence du mal, au sens religieux. presque théologique, du mot. Je pense aux jeunes garçons de David Sterne, bien entendu, mais aussi à plusieurs autres de vos personnages qui vivent le bien et le mal comme des absolus, sous le regard d'un Dieu terrible, impitovable. On s'étonne de lire des choses comme celles-là dans vos romans des années soixante, alors que chez presque tous les autres romanciers québécois de la même période sauf peut-être Réjean Ducharme - la question religieuse, la question de l'absolu semble s'être totalement dissipée. Dieu n'est-il pour vous, dans ces romans, que la figure mythique d'une Grande Terreur, ou bien son nom signifie-t-il autre chose?

M.-C.B.: Je pense qu'à l'époque, durant les années soixante, tel que je l'écrivais à ce moment-là, c'était peut-être plus une terreur morale 
que religieuse, c'est-à-dire la terreur d'une dictature morale. Que je redoute toujours d'ailleurs... Je pense que Ducharme a une ironie devant cette dictature morale, que je n'ai pas eue. Mais... c'est peut-être lui qui a raison. Parce que, en fait, surtout dans les années soixante, soixante-cinq, soixante-dix, il faut peut-être considérer que tout ca s'est écrit bien jeune, avec tous les défauts de la jeunesse...

V.I.: $\quad$..les qualités, aussi.

M.-C. B.: ...donc il n'y a pas d'ironie quand on est très jeune. Je ne sais pas si vous avez remarqué...

V.I.: J'ai remarqué!

V.I.: Vous et Réjean Ducharme avez beaucoup d'amitié l'une pour l'autre. Vous vous êtes dédicacé des livres: de votre côté, Manuscrits de Pauline Archange: du sien, I'Océantume. Comment le voyez-vous. Réjean Ducharme?

M.-C. B.: Je le vois, avec vous, comme un grand écrivain. Je pense que c'est un garçon remarquable, très isolé dans notre littérature, un peu comme Aquin. Aussi isolé que ça, disons, parce que, vraiment, il ne veut pas plaire...

V.I.: Sûrement pas I

M. -C. B.: Non. II est très anarchiste et très seul.

V.I.: Est-ce que vous voyez une parenté entre ce qu'il écrit et ce que vous écrivez?

M.-C. B.: C'est un homme d'images, un visionnaire. Là-dessus, on peut être proches...

V.I.: Mais sur le plan moral en quelque sorte?...

M.-C. B.: C'est un moraliste, je ne dirais pas plus dégagé, mais... plus imagé. Comme dans sa vie, comme dans son ètre, c'est un garçon qui part beaucoup, il s'efface. Donc, dans son ceuvre, on le sent absent, on sent qu'il est lui-même en voyage, il part dans ses personnages. Ce qu'on ne sent pas chez moi. On sent un peu trop te lien avec la réalité.

V.I.: Ducharme est infiniment plus lyrique. La question morale, chez vous, s'exprime de façon plus évidente...

M.-C. B.: Je pense.

V. I.: Tout se passe, dans certains de vos romans tout au moins, comme si le salut venait du mal; comme si la connaissance, l'expérience du mal, le crime même, étaient la seule voie d'accès à ce que le «mauvais prêtre. Benjamin Robert, dans Vivre! Vivre!, appelle la "sainteté ». Je le cite: "si nous avions la force de condamner la vie. 
de l'aimer d'un amour dévastateur et sans pitıé, tel cet amour maudit qui consume l'âme du véritable meurtrier, amour dont il pourrait s'éprendre jusqu'à la sainteté, nous aurions le secret d'une autre vie dont l'absolue sécheresse. l'absolue vérité nous éblouiraient. Comment relisez-vous cela aujourd'hui?

M. -C. B.: Je pense que ce sont des vérités dostoievskiennes. Parce que ce sont vraiment des choses qui reviennent beaucoup dans les Possédés. Et moi, j'étais, enfin, j'étais, je peux pas le renier, je suis toujours très touchée par les extrêmes chez les gens. Je pense que Benjamin Robert n'était pas un mauvais prêtre, voyez-vous, parce qu'il aimait tellement les autres. II y a quelques failles sexuelles chez lui, mais disons que ca fait partie de l'amour des autres, tout ca, pour moi. Je n'ai pas tellement de jugement moral devant la générosité.

V.l.: Vous avez parlé de Dostoïevski. En vous lisant parfois il peut nous arriver de penser aussi à Bernanos, à Graham Greene. Est-ce que ces ceuvres-là vous ont touchée?

M.-C. B. : Ca m'a toujours beaucoup touchée, parce qu'ils sont tous deux émus par la fragilité de l'homme, celle qui se trouve dans un état de pouvoir faiblissant, mais aussi la fragilité de l'homme ordinaire.

L'art, la beauté

Andantino. Ce mouvement était long et contrastant. Jouez, Johann. Les planètes s'animent... Jouez, Johan. Tout est immortel et rien ne sera oublié de ce qui fut.

les Voyageurs sacrés

Mais Geneviève pensait que l'art seul, en ce monde, nous permet ainsi d'investir toute notre liberté et que chacun peut reconnaître là que sa douleur a lui est aussi universelle que l'universelle conception qu'un sculpteur génial en a eue. Aucun tribunal, aucune société ne peuvent ici défendre même à un meurtrier d'avoir l'illusion qu'il est devenu bon au contact d'une ceuvre belle, car cet espoir n'appartient qu'à lui.

les Nuits de l'Underground

V.I.: Est-ce que je me trompe en pensant que la préoccupation morale au sens où nous venons d'en parler, ou la préoccupation du salut. s'éloigne progressivement dans vos derniers romans? Dans le Loup par exemple, le narrateur parle de ucompassion», de acharité», de a rédemption. - ce qui d'ailleurs, dit-il, irrite fortement ses amis -. mais au début des Nuits de l'Underground c'est d'autre chose qu'il est question: « L'amour de Geneviève Aurès pour Lali Dorman naquit comme une passion pour une cuvre d'art». Peut-on parler d'une 
évolution, d'un changement de perspective? Est-ce que l'art, pour vous, participe d'un absolu?

M.-C. B.: Sans doute qu il participe d'un absolu. Enfin, pour moi, il est essentiel à ma vie. Mais, naturellement. je ne peux pas dire si... Je pense que les préoccupations morales que j'avais plus jeune deviennent plus paiennes disons. simplement au niveau humain, quoi. Tout à coup. on se dit qu'on ne peut plus juger les autres, on ne peut pas se juger soi-même. C'est très ingrat d'avoir une morale quelconque. c'est difficile... L'art n'en a pas. Quand on voit une ceuvre d'art, un Courbet. il n'y en a pas. il n'y a pas de... Il y a la peinture humaine. c'est tout.

V.I.: Alors, l'art échappe à la préoccupation morale, et c'est pour ca qu il vous est précieux?

M.-C. B.: Aussi, il définit quelque chose qui va rester, il garde un moment de vie intact.

V.1.: Vous parlez souvent de la Beauté, dans vos premiers romans comme dans les derniers, et il me semble qu'elle peut avoir deux sens: un sens de vie, et un sens de mort. Je lis cela, par exemple, dans un livre qui est à mi-chemin du poème et du roman. les Voyageurs sacrés.

M.-C. B.: Mais là, encore une fois, c'est un récit d'extrême jeunesse.

\section{V.I.: Ce n'est pas si loinl}

M.-C. B.: C'est quand même assez loin. C'est tout autour de la question de l'art, et de l'art le plus généreux, le plus spontané, la musique, que j'envie beaucoup. Je veux dire que c'est un art, comparé aux autres... un art de délices quoi. Peut-être que je me trompe... Mais c'était autour de cette question: je trouve admirable qu'on soit transporté ailleurs. On est transporté ailleurs par la musique - mais aussi par les personnages des Voyageurs sacrés qui vivent dans un monde où. quand même, tout semble pour moi exquis, facile...

V.I.: Une sorte d'évasion?

M.-C. B.: Oui. Bien sûr, le texte est difficile parce qu'il y a un déchirement amoureux entre les êtres, triangulaire. Mais aujourd'hui, je ne sais pas, j'aurais une toute autre vision de ca.

\section{V.I.: Et qui serait?}

M.-C. B.: Bien, d'abord, évidemment la musique dont il est question, c'est la musique classique. Aujourd'hui le son, la musique sont remis en question.

\section{I.: Comme la littérature?}

M.-C. B.: Oui. Bien sûr, ce serait plus chaotique, mon texte serait plus chaotique. 
V.I.: Ce serait une évasion moins paisible!

M.C. B.: Oui, c'est ca, je pense que c'est un texte d'adolescence dans le sens où c'est une évasion.

V. I.: II y a aussi dans les Voyageurs sacrés l'échec de la pièce de Miguel. qui est suivie de son suicide. II me semble qu'à ce moment-là Miguel se trouve devant un défi de la Beauté, et que la Beauté le tue...

M.-C. B.: Bien sûr, c'étaient des idées que j'avais, que la Beauté nous tuait, que la fin de la jeunesse nous tuait. Maintenant, je me dis que c'est le contraire. Parce qu'on commence à vivre quand on commence à réfléchir, et comme on ne réfiéchit pas à cet âge-là, on se laisse emporter... Est-ce qu'on est juste, est-ce qu'on a une pensée claire? Je pense que c'est un texte qui a beaucoup d'exaltation mais peu de résonance intérieure. C'est moi qui le dis, hein?

La saison d'Emmanuel

Pivoine retourna à la terre sans se plaindre et moi j'en sortis en criant. Mais non seulement. je criais. mais ma mère criait elle aussi de douleur. et pour recouvrir nos cris, mon père égorgeait joyeusement un cochon dans l'établel Quelle journée! Le sang coulait en abondance, et dans sa petite boite noire sous la terre. Pivoine (JosephAimé) dormait paisiblement et ne se souvenait plus de nous.

- Un ange de plus dans le ciel, dit Monsieur le Curé. Dieu vous aime pour vous punir comme cal

Ma mere hocha la tête: en une année.

- Mais Monsieur le Curé, c'est le deuxième

- Anl Comme Dieu vous récompense, dit Monsieur le Curé.

Monsieur le Curé m'a admiré dès ce jour-là. La récompense, c'était moi.

Une saison dans la vie d'Emmanuel

V.I.: Quand nous passons de l'Insoumise...

M.-C. B.: c'est plus sérieux, la.

V.I.: $\quad$... le roman qui précède tout juste Une saison dans la vie d'Emmanuel, a ce dernier roman, on note un changement assez extraordinaire. C'est bien le mème écrivain qu'on lit, on reconnait des thèmes, certaines façons d'écrire, mais ce sont surtout les différences qui sautent aux yeux. Au lieu d'une méditation passionnée, lyrique. 
sur la solitude, la mort, les rapports entre les êtres, on se trouve en présence d'un récit extrêmement vif, cruel, mais drôle aussi, souvent. qui semble - je parle d'une première impression de lecture beaucoup plus "réaliste" que les précédents. Comme si, pour la première fois, Marie-Claire Blais écrivait un roman vraiment québécois, vraiment "enraciné" comme on dit. Est-ce que vous avez voulu écrire un roman "réaliste"... dans une certaine mesure?

M.-C. B.: C'est étrange, parce que pas du tout, mais vraiment pas du tout. C'est un roman qui a été écrit aux Etats-Unis, et sans aucune intention réaliste. Evidemment, pour moi, c'est une cauvre de fiction, ça le demeure. Avec quelque chose d'international, c'est-à-dire qu'on peut y retrouver sa souffrance partout, en Argentine ou ailleurs. La souffrance des familles, des groupes humiliés, peut-être opprimés.

V.I.: Mais dans la forme, c'est très différent de ce que vous avez écrit auparavant. et mème de ce que vous allez écrire par la suite.

M.-C.B.: Oui. C'est difficile pour moi de parler de la forme, parce que... La forme me semble assez joyeuse.

V.I.: Ca bouge énormément, il y a de l'action, de la fantaisie, et même de la drôlerie comme vous le dites.

M.-C.B.: II y a certainement un sens de l'humour qui commence à naître à ce moment. Et les personnages ont une distance envers eux-mêmes, comme Jean-Le Maigre a une distance envers lui-même et ses frères. Ca devient plus fort. ca devient plus riche dans la texture.

V.I.: Je voudrais vous demander de me parler de Grand-Mère Antoinette. C'est un des personnages, je pense, les plus étonnants du roman. C'est votre première grand-mère. Elle paraît dure au premier abord. elle gronde sans cesse les enfants, mais elle est aussi leur plus sûr refuge, elle est la seule à soutenir vraiment Jean-Le Maigre dans ses entreprises d'écriture. Elle est le personnage le plus traditionnel. et en même temps le plus ouvert à ce qui vient, c'est-à-dire aux enfants, à ce qu'ils vivent. Si je vous demandais d'être à la fois la lectrice et l'auteur de votre roman, comment en parleriez-vous?

M.-C. B.: Ce que vous dites, c'est très juste, c'est une très bonne description, c'est-à-dire qu'elle est une fermme en apparence austère, fermée - et très généreuse. Elle est très généreuse avec Jean-Le Maigre. Evidemment, elle a a la fois l'amour et l'autorité, ce qui est toujours dur dans les situations affectives. Mais elle a l'amour aussi, plus que l'autorité, disons. Elle a le sens critique déplorable, mais elle a tant d'amour et de générosité, et je dirais de compassion, que malgré son ignorance qui est grande, elle finira par accepter Jean-Le Maigre et comme être humain et comme poète, je pense. Même si elle le nie: enfin... C'est curieux, parce que ce sont des émotions contraires, mais... 
V.l.: Oui, c'est précisément ce qui est très beau dans ce personnage, la conjonction d'èléments opposés.

M.-C. B.: II avait besoin d'une femme comme ça dans sa vie.

V.I.: Mais par opposition à Grand-Mère Antoinette, les parents immédiats, le père et la mère, n'ont pas beaucoup de présence dans Une saison. Dans votre cuvre d'ailleurs, de façon générale, les parents, les adultes, ne sont jamais très brillants.

M.-C. B.: Moi, personnellement, j'ai beaucoup d'admiration pour mes propres parents. Mais je pense que dans les livres on peut se permettre d'être injurieux envers ce que vous appelez les adultes. Réjean décrit ca admirabtement. C'est vraiment des juges envers les autres. Alors. quand l'adulte devient exécrable, pour moi il est comme un adulte dans l'œuvre de Kafka...

V.I.: II devient tout à fait exécrable!

M.-C. B.: Tout à fait exécrable et dangereux.

V.I.: $\quad$ II y a des adultes plus ambigus dans votre ceuvre. Je pense en particulier aux mères qui sont comédiennes, la mère de Tête Blanche, la Marie-Christine du Jour est noir. Vous semblez leur faire un sort tout à fait spécial, précisément parce qu'elles sont comédiennes.

M. -C. B.: Peut-être parce que c'est la vie de bohème qui m'attirait à l'époque et qui m'attire toujours. C'est-à-dire ce côté, ce rôle nomade... de l'amour, aussi. De crampes, d'être de passage...

V.I.: Mais vous êtes très dure pour ces personnages-là.

M.-C. B.: Peut-être que je l'étais, mais je ne le serais plus!

V.I.: II y a aussi un autre personnage d'adulte, femme, qui me frappe beaucoup dans la trilogie de Pauline Archange, c'est Germaine Léonard. C'est une femme sympathique au départ, elle a de la maturité, elle est intelligente, elle est prête à aider les jeunes, en particulier Pauline Archange, mais elle finit par décevoir.

M.-C.B.: Je pense que ca, c'est un personnage de grande dimension. Je ne l'ai pas bien décrit dans mon travail, mais c'est un personnage de grande dimension, auquel j'aimerais beaucoup revenir. Parce que c'est la femme, bon, c'est un peu aujourd'hui l'assistante sociale...

V.I.: $\quad$... la femme libérée?

M. -C. B. : . . la sociologue, mais c'est un ètre qui a comme idéal d'être présente aux autres. Même à l'époque où je la décris. c'est la femme libérée, la femme généreuse, la femme libérée dans le sens le plus vaste. Libératrice aussi.

V.I.: Mais dans votre roman, elle déçoit profondément Pauline Archange. 
M.-C. B.: Parce que Pauline Archange n'a que huit ou dix ans, et qu'elle n'est pas capable d'absorber encore. Mais je pense que. comme caractère, c'était un caractère intéressant.

V.I.: II faut quand même en venir au Prix Médicis... Votre premier roman, la Belle Bête. avait été publié en France, chez Flammarion, et il avait reçu un accueil assez discret, si je me souviens bien. Puis, quelques années et quelques livres plus tard, avec Une saison, c'est le succès, la célébrité. Vous avez été étonnée?

M.-C. B.: II faut dire que dans le temps de la Belle Bête, à Paris, ce qui était choquant chez moi, c'est que j'étais d'une discrétion absolue... Je suis contre la discrétion maintenant! J'étais un être impossible. très sauvage. Mais j'avais un ami qui était merveilleux. Pasteur Vallery-Radot, vous l'avez peut-ètre connu à l'époque...

V. I.: Non.

M.-C. B.: C'était un être extraordinaire, qui m'avait beaucoup aidée. II est mort maintenant. II m'avait beaucoup aidée à présenter ce livre, mais je ne l'ai pas assisté si je puis dire.

V.I.: C'est lui qui avait présenté le livre chez Flammarion?

M.-C. B.: Non, il m'avait aidée dans le sens qu'il avait écrit un très beau papier dans le Monde, enfin dans le Figaro, je ne sais plus... J'avais une terreur de tout ça à l'époque, si bien que c'est vrai qu'il y a eu un étonnement plus tard. Quand je suis revenue plus tard, j'étais plus fortel

V.I.: Est-ce que c'était très important pour vous d'atteindre le public français, le public international?

M.-C. B.: C'est très important aujourd'hui. Je ne suis pas sûre que ce l'était à l'époque. Tout m'arrivait un peu par hasard. Je n'avais pas les préoccupations que j'ai aujourd'hui de communiquer beaucoup avec les Français, avec l'Europe. Dans un sens, oui, le succes parisien ca vous frappe, pas comme une catastrophe malheureuse, mais... comme une catastrophe heureuse!

V.I.: La critique... Si je me souviens bien, Une saison dans la vie d'Emmanuel avait recu au Québec un accueil moins enthousiaste qu'en France. Est-ce que vous avez eu l'impression d'être comprise, là-bas?

M.-C. B.: II ne faut pas être injuste, parce que je pense que Monsieur Jean Ethier-Blais avait été très gentil. Puis quelques autres... A l'époque. je ne lisais pas les critiques. Mais il me semble que ca n'avait pas été trop mal reçu. Avec réticence, disons. La réticence, chez nous, est visible dans la critique... C'avait été bien reçu en France, mais j'ai peur parfois... Cet accueil n'était-il pas lié à quelque chose de rural, de local, de folklorique encore... 
V.I.: $\quad$... le còté "réaliste» que vous n'aviez pas voulu...

M.-C. B.: ... que je n'ai pas, et que je n'aurai jamais. Pas plus que Réjean Ducharme. Je pense que nous sommes des témoins de notre temps. Errants, assez nomades.

V.I.: Vous n'êtes pas des écrivains québécois avant tout...

M.-C. B.: Nous sommes des écrivains québécois, par tradition naturellement. par amour. Mais ni lui ni moi n'aimons les étiquettes, je crois.

V.I.: J'ai l'impression que la rencontre avec la France, l'expérience de la France, ont été trés importantes pour vous. Vous y avez vécu - vous avez fait un assez long séjour en Bretagne, après le Prix Médicis. si je ne me trompe. Et vous avez. dans Une liaison parisienne, parlé de la France, des milieux littéraires parisiens, d'une facon caustique. Est-ce un roman à clés, comme certains l'ont pensé?

M. -C. B.: Je ne sais pas... parce que j'ai beaucoup de critiques à l'égard de ce livre. Mais en tout cas, ce qui est sûr, c'est qu'il y a beaucoup de choses vraies. Sous une forme maladroite, il y a beaucoup de choses vraies sur la France, et que critique aussi Monsieur Romain Gary dans ce livre dont je vous parlais. C'est très vrai qu'il y a un parisianisme destructeur. Maintenant, nous sommes liés aux vrais Français, nous aimons la France, et c'est tout. Je serais peut-être moins acerbe aujourd'hui, parce qu'une humiliation dans un monde de critiques littéraires ou de cliques comme ca, c'est très dur à prendrè, mais quand on connait le peuple français - maintenant je le connais bien, je pense - on est attach6́, et ceci disparait, enfin c'est comme un nuage, un mauvais nuage.

V.I.: Vous avez un bon souvenir de ces sejours, maintenant?

M.-C. B.: Moi, j'ai beaucoup de nostalgie de la France, j'y retourne tout le temps. Je suis très liée...

V.I.: Est-ce qu'il y a, pour vous, une image privilégiée de la France?

M.-C. B.: Maintenant, ce qui m'intéresse par rapport à la France, c'est l'écrivain de qualité en France, qui est aussi ignoré, aussi mal traité. que... les nôtres chez eux peut-être, et qui souffre des mêmes abandons, des mèmes oublis. et de la part des critiques et de la part des... II souffre des mèmes folklorismes, du même décor, là, faux, du même bavardage que nous. Enfin, l'écrivain de qualité souffre autant là-bas que nous quand nous sommes publiés chez eux - nous souffrons beaucoup. Je suis sûre qu'un écrivain trançais de qualité n'a que très peu de dialogue... C'est ca qui m'intéresse maintenant, c'est de rencontrer des écrivains français pleins de cœur, pleins de sang. pleins de saveur, qui correspondent un peu à ma pensée. Et j'en lis de temps en temps, qui sont très beaux, et dont la critique ne parle pas. 
V.I.: Est-ce que vous avez beaucoup d'amis parmi les écrivains français? M.-C. B. : J'ai quelques amis, mais je suis... évidemment, je suis très timide. V.I.: Vous lisez des romans actuels.

M.-C. B.: Oui. Mais j’ai quelques amis aussi.

V.I.: Revenons à l'ordre chronologique... Il y a une chose qui m'a étonné, et qui a sans doute étonné beaucoup de vos lecteurs, c'est qu'après Une saison dans la vie d'Emmanuel, le Prix Médicis, vous semblez changer de cap. soudainement. Vous auriez pu exploiter votre succès. écrire de nouveaux romans qui ressembleraient à Une saison. D'ailleurs vous avez publié, dans les Lettres nouvelles et Liberté, deux textes où l'on retrouve la même forme d'écriture, voire les mêmes personnages. Mais vous ne poursuivez pas. De quoi s'agit-il? D'un refus conscient, déterminé?

M.-C. B.: On ne sait jamais. On ne peut pas dire que ce soit si conscient que ça. Peut-être, dans l'avenir, ce sera autrement. Mais c'est sūr que moi je ne veux pas répéter quelque chose. J'en suis absolument incapable. Mon travail, c'est une recherche, je m'aventure à mes propres risques et périls. C'est une aventure, et dans le style, dans la forme, et dans la recherche humaine, je veux aller le plus loin possible. Donc, je ne voudrais pas rester accrochée à Une saison. pas du tout. Vous le savez vous-même, quand on a fini une œuvre on va vers une autre. C'était comme ca, c'était achevé. Pourquoi retourner dans quelque chose d'achevé? On grandit, on grandit beaucoup après avoir fait un travail comme ca, et on veut aller ailleurs.

V.I.: Vous avez dit que vous réserviez l'avenir. Est-ce que ca signifie que pour vous le retour à une forme d'expression semblable à celle d'Une saison n'est pas exclu?

M.-C. B.: C'est difficile, parce que je suis tout à fait dans une autre recherche, maintenant, je suis trés loin d'Une saison. Même. je trouve que c'est accaparant, parce que ca empêche les autres livres de vivre. Les autres que j'ai écrits sont un peu a l'écart a cause d'Une saison. IIs sont peut-être plus remarquables I

Mémoire du passé. mémoire du présent

Je rêvais tant d'écrire la vie que je croyais parfois la posséder; mais quand je voulais écrire ces choses du passé, elles semblaient disparaitre dans la brume, ne laissant devant moi sur la page blanche, dans un cahier écorché par l'usure, que la brève silhouette d'un être que j'avais pourtant longtemps aimé: son dos humilié s'engouffrait dans une rue, s'éloignait, et songeant plus tard à cette disparition, j'avais soudain l'âme remplie de terreur et de respect... 
V.I.: Donc, tout de suite après Une saison, vous plongez, vous vous enfoncez dans ce grand roman de la mémoire qu'est la trilogie de Pauline Archange. Ce n'est pas sans raison qu'on pense à Proust en lisant la première phrase du roman: aComme le chceur de mes lointaines misères, vieilles ironies que le temps a revètues du sourire de la pitié. (mais une pitié puant légèrement la mort) les vieilles religieuses, qui, autrefois, berçaient ma vie de leur cruelle bonté...», et catera. Ce changement de ton, d'écriture, vous était sans doute nécessaire. II ne venait pas seulement d'une lecture - ou d'une relecture - de Proust... A quelle nécessité cela répondait-il?

M.-C. B.: Je suis encore très plongée dans la mémoire. Disons que c'est quelque chose qui me persécute... Ca, je pense que c'est un livre auquel je retournerai peut-ètre. Une dernière exploration - enfin, disons qu'on s'accorde quelques années. Je veux dire une dernière exploration de ce thème-là, de Pauline, la société québécoise de l'époque que je décris, qui grandit avec l'auteur. Ca m'intéresserait d'y retourner avec plus de maturité; parce que je considère qu'il y a encore des failles là... Sans doute que je le ferai, je me dis que ce n'est pas impossible. Mais la mémoire que j'ai maintenant. elle est plutôt une mémoire du présent...

\section{V.I.: Qu'est-ce que vous entendez par là?}

M.-C. B.: C'est-à-dire que je me sens très proche de.... je me sens très nerveuse. excédée par tout ce qui se passe aujourd'hui, aujourd'hui mème, là, et je suis un être curieux. avide de tout ce qui se passe aujourd'hui, de tout ce que je vis aujourd'hui, dans l'instant présent. Comme avec vous, la... Mais j'ai besoin de capter la mémoire du présent. A cause de ca, peut-être que je n'irai pas tout de suite peut-être plus tard - vers l'exploration de ce passé, qui est intéressant, qui est riche, qui est plein de détails. Je suis en ce moment dans l'angoisse du présent, comme vous peut-être, dans la hantise du présent, dans le halètement du présent, tout ce que ca comporte pour nous d'être vivants. C'est tout ce qui m'importe pour l'instant. Peut-être, plus tard...

V.I.: Quand vous parlez de la hantise du présent, est-ce que vous parlez en particulier de la situation du Québec. de toutes les questions qui s'agitent ici, au Québec, à propos de notre situation collective? Est-ce que vous êtes politisée?

M.-C. B.: Je suis pas sûre d'être politisée, mais je suis sûre d’être très attentive a ce qui arrive en ce moment. Je pense que nous sommes très troublés en ce moment, nous sommes une partie... haletante de cet univers haletant... Et ce qui nous arrive au Québec, c'est un isolement culturel qui pourrait ètre très grave pour notre culture et notre culture politique et sociale, je pense...

\section{V.I.: Comment décririez-vous cet isolement culturel?}


M.-C.B.: Disons que j'ai une certaine inquiétude pour notre avenir, en ce moment. Je suis très attentive, je me dis que nous sommes très jeunes, très vivants, très vibrants, très réceptifs...

V.I.: Est-ce qu'en parlant d'isolement, vous désignez une tendance que nous aurions à nous refermer un peu trop sur nous-mêmes?

M.-C. B.: Nous avons toujours fait ça. Peut-être que maintenant nous allons être plus attentifs à ça, à nous ouvrir davantage à une culture universelle. Mais c'est quand même beaucoup rêver, peut-être, de dire ca.

V.I.: Vous n'habitez pas Montréal, vous vivez dans les Cantons de l'Est, mais vous rencontrez beaucoup les écrivains d'ici, en particulier les écrivains féminins, si je ne me trompe pas. Vous participez aux mouvements féministes?

M.-C. B.: Bien sũr. Mais ca, j'y participais plus encore aux États-Unis. J'ai toujours été là-dedans. Mais je suis quand même quelqu'un d'assez solitaire.

V.I.: Justement, si nous parlions un peu de votre expérience américaine. Vous avez passé combien d'années aux Etats-Unis, à la suite de votre rencontre avec le critique Edmund Wilson?

M. -C. B.: Sept ans.

V.I.: A Wellfleet, Cape Cod?

M. -C. B.: Oui.

V.I.: Et qu'est-ce que c'était, Wellfleet, pour vous?

M.-C. B.: Bien, c'était intéressant, parce que c'était un milieu très culturel. Enfin, pour une fille dans la vingtaine c'était un enrichissement que je n'aurais pas connu ailleurs, je pense. C'était culturellement très intéressant, très vivant. très riche. II $y$ avait Edmund Wilson. il y avait des artistes - et peut-être que c'est là que j'ai découvert mon amour pour la peinture. C'est sûr que c'a été énorme pour moi.

V.I.: Est-ce que vous êtes devenue... un peu américaine?

M.-C. B.: Non. Non. Mais j'étais liée à des féministes et à des pacifistes américaines. Tout cela $\mathrm{m}$ 'a énormément enrichie à un moment où peut-être je n'étais mème pas capable de le prendre, disons. Aujourd hui, je le reçois.

L'écriture, la solitude. le bonheur

II s'approcha de la fenêtre givrée et il me sourit. II y avait dans ce sourire une telle amitié, une si tendre vaillance que je sentis soudain renaitre mon courage et, emportant cette vision dans la tempête, je courais en pensant avec joie: «C'est lui... l'ange de Dürer, je l'ai vu, enfin!. 
V.I.: Nous avons commencé cet entretien en parlant de l'écriture. Nous le terminerons en ... en parlant encore, si vous voulez. Il y a beaucoup de personnages d'écrivains dans vos romans. Il y a le Tête blanche du roman du mème nom, le Josué du Jour est noir, beaucoup d'autres, et ce sont tous des ètres qui, convaincus de la très grande importance de ce qu'ils font, ont aussi des inquiétudes profondes. qui ont de la difficulté à établir un contact avec le milieu où ils vivent. Est-ce que pour vous tout écrivain véritable, authentique, ne serait pas de quelque manière un aécrivain maudit *?

M.-C. B.: Je pense, oui... Je sais pas si vous ètes d'accord avec moi. C'est certainement, comme disait Conrad, un $\propto$ outcast $*$, c'est certain... Et dans notre société qui est constamment portée vers les choses matérielles, c'est un être spirituel, l'écrivain est un ètre spirituel, qui a des valeurs spirituelles... II peut ètre matérialiste, aussi, heureusement I Mais je pense qu'il a un grand isolement devant lui.

V.I.: Vous avez parlé de votre timidité, mais en dehors de toute question personnelle est-ce que vous ne définiriez pas la condition de l'écrivain par l'isolement? Par la solitude acceptée, nécessaire?... C'est du moins ce que je crois lire dans vos livres.

M.-C. B.: Oui, elle est acceptée, et elle est nécessaire, puis elle est volontaire, parce qu'on s'isole pour travailler, on va à la campagne pour travailler. où on ne peut pas rencontrer les autres... Peut-être qu'on a de grandes périodes, après, où on est très frivole, mais en général la période de travail est très concentrée, très réfléchie.

V. I.: Si je ne me trompe pas, vous étes très disciplinée dans votre travail.

M.-C. B.: Ce qui est terrible dans ma nature, c'est que je suis très disciplinée dans mon travail, et peu dans ma vie. Alors... C'est toujours en conflit. Mais ca ne fait rien...

V. I.: Est-ce que vous êtes heureuse?

M.-C. B.: Bien, j'essaie, je crois beaucoup a l'artiste heureux. Je pense qu'il faut qu'il le soit. Je voudrais que les autres le soient, en tout cas. le deviennent, parce que je pense que les choses qu'on a connues quand on était très jeune, ce sentiment justement de ne pas faire partie de la société, d'être un peu un enfant maudit, on souhaiterait que ceux qui viendront ne connaissent plus ca. Que ce soit une nouvelle ère. L'ère de l'artiste heureux, c'est pas impensable...

V.I.: Est-ce cela que vous poursuivez dans votre travail?

M.-C. B.: Dans mon travail personnel d'écriture, c'est toujours extrêmement douloureux. Pas seulement pour moi, je pense que pour tous les autres aussi... Ca sera toujours douloureux, mais c'est un rayonnement ce qu'on fait, malgré tout le malheur qu'on décrit, malgré tout le malheur sur lequel on pose les doigts, cette vision du monde funeste... II y a quand même en nous une sérénité, je pense. pas 
seulement chez moi, chez Réjean, chez Anne Hébert. chez Langevin. chez d'autres. II y a une espèce de sérénité de fond. Autrement, nous ne pourrions pas travailler.

V.I.: Et vous écrivez, présentement?

M.-C. B.: Oui.

V.I.: Est-il indiscret de vous demander ce que vous faites?

M.-C. B.: C'est encore une fois une vision très présente et peut-être très pesante aussi mais j'essaie de la rendre légère, légère dans l'écriture du moins - de notre monde, des jeunes gens entre treize ans et vingt-cinq, de la vision qu'eux ont de notre monde. Et notre monde. il est comme nous l'avons fait, comme nous le faisons tous les jours. lls en sont les témoins... blessés. 